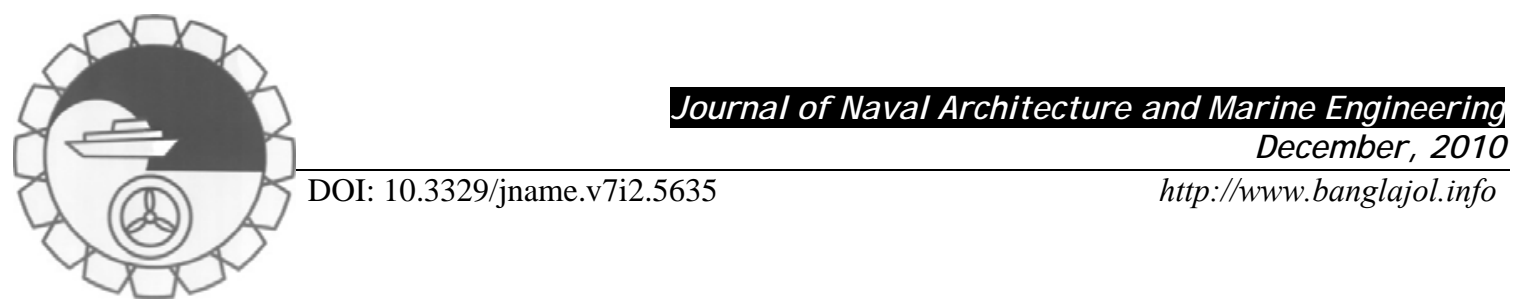

\title{
CRITICAL BEHAMOUR OF THE MHD FLOW IN CONVERGENT-DIVERGENT CHANNELS
}

\section{S. Alam ${ }^{1}$ and M.A.H. Khan ${ }^{2}$}

${ }^{1}$ Department of Mathematics, Jagannath University, Dhaka-1100, Bangladesh, Email: sarwar.alamuiu@gmail.com
${ }^{2}$ Department of Mathematics, Bangladesh University of Engineering and Technology, Dhaka-1000, Bangladesh
Email: mahkhan@math.buet.ac.bd
Abstract:
The effect of external Magnetohydrodynamic (MHD) field on the steady two-dimensional nonlinear
flow through Convergent-Divergent Channels of a viscous incompressible electrically conducting
fluid is investigated. We compute the critical behaviour of the solution govern by the equation. Our
approach uses the power series in order to observe the instability of the problem. The series is then
summed by using various generalizations of the Pade'-Hermite approximants. The critical values of
various parameters and type of the principal singularity are found for different choice of MHD effect.

Keywords: Magnetohydrodynamic, Critical behaviour, Convergent-Divergent channels, Bifurcation, Approximation method.

$$
\begin{array}{cl}
\sigma_{\mathrm{e}} & \text { Conductivity of the fluid } \\
\alpha_{\mathrm{c}} & \text { Critical values of Channel angular width } \\
\operatorname{Re}_{\mathrm{c}} & \text { Critical values of Reynolds number } \\
\beta_{\mathrm{c}} & \text { Critical exponent } \\
\rho & \text { Density of the fluid } \\
\mathrm{B}_{0} & \text { Electromagnetic induction } \\
\mathrm{H}_{0} & \text { Intensity of Magnetic field } \\
\mathrm{V} & \text { Tangential velocity component }
\end{array}
$$
v
Kinematics viscosity coefficient
$\alpha \quad$ Magnitude of channel angle
Ha Magnetic parameter
$\mu_{\mathrm{e}} \quad$ Magnetic permeability
Q Rate of volumetric flow
$\mathrm{u} \quad$ Radial velocity component
G Stream function
HODA High-order Differential Approximant

\section{Introduction}

The MHD flow of a viscous electrically conducting fluid through Convergent-Divergent Channels has remarkable assistance in developing the mathematical model of several industrial and biological systems. Various applications of this type of mathematical model are in understanding the flow of rivers and canals, and the blood flow in the human body. Meanwhile, practically the magnetic field has a significant effect in the fluid flow through Convergent-Divergent Channels. Jeffery and Hamel (1915-16) first studied the two-dimensional steady motion of a viscous fluid through Convergent-Divergent Channels which is called classical JefferyHamel flow in fluid dynamics. Fraenkel (1962) then investigated the laminar flow in symmetrical Channels with slightly curved walls. In his analysis the velocity field of the flow was obtained as a power series in small curvature parameter where the leading term is the Jeffery-Hamel solution. Sobey and Drazin (1986) studied some instabilities and bifurcations of two-dimensional Jeffery-Hamel flows using analytical, numerical and experimental methods. Banks et al. (1988) extended the analysis of perturbation theory of pitchfork bifurcation of the Jeffery-Hamel flows and used as a basis to investigate the spatial development of arbitrary small steady two-dimensional perturbations of Jeffery-Hamel flow both linearly and nonlinearly for nearly plane walls. They found that there is a strong communication between the disturbances up and downstream when the angle between the planes exceeds a critical value, which depends on the value of the Reynolds number. Moreover, the steady flow of a viscous incompressible fluid in a slightly asymmetrical channel was studied by Makinde (1997). He expanded the solution into a Taylor series with respect to the Reynolds number and performed a bifurcation study using Drazin-Tourigny method (Khan et al., 2003). Makinde (2006) investigated the Magnetohydrodynamic (MHD) flows in Convergent-Divergent Channels which was an extension of the classical Jeffery-Hamel flows to MHD. He interpreted that the effect of external magnetic field works as a parameter in solution of the MHD flows in Convergent-Divergent Channels. Therefore, a non-dimensional 1813-8235 (Print), 2070-8998 (Online) ( 2010 ANAME Publication. All rights reserved. $\quad$ Received on: July 2010 
magnetic parameter $\mathrm{Ha}$ was involved with the flow Reynolds number and the Channel angular width. A Perturbation series of twenty-four terms in powers of parameters $R e, \alpha$, and $H a$ was obtained by Makinde (2006) and showed how the flows change and bifurcate as the flow parameters vary by using algebraic approximate method.

Kayvan et al. (2007) analysed the applicability of magnetic fields for controlling hydrodynamic separation in Jeffrey-Hamel flows of viscoelastic fluids. Assuming a purely symmetrical radial flow, they obtained a thirdorder nonlinear ODE as the single equation governing the MHD flow of this particular fluid in flow through converging/diverging channels by similarity analysis. With three physical boundary conditions available, they used Chebyshev collocation-point method to solve this ODE numerically. The effect of magnetic field was found to be more striking in that it is predicted to force fluid elements near the wall to exceed centerline velocity in converging channels and to suppress separation in diverging channels. Interestingly, the effect of the magnetic field in delaying flow separation is predicted to become more pronounced the higher the fluid's elasticity.

However, a numerical investigation of the effect of arbitrary magnetic Reynolds number on steady flow of an incompressible conducting viscous liquid in convergent-divergent channels under MHD was presented in O.D. Makinde (2008). He solved the non-linear 2D Navier-Stokes equations modeling the flow field using a perturbation technique applying the special type of Pade'-Hermite approximation method implemented numerically on MAPLE and a bifurcation study was also performed. The increasing values of magnetic Reynolds number cause a general decrease in the fluid velocity around the central region of the channel. The flow reversal control is also observed by increasing magnetic field intensity. The bifurcation study reveals the solution branches and turning points.

Our work illustrates the comparison with Makinde(2006) about the effect of magnetic field on two-dimensional, steady, nonlinear flow of a viscous incompressible conducting fluid in Convergent and Divergent Channels. The critical relationship among the flow parameters have not been studied yet, according to the author's best knowledge. The non-dimensional equation considering magnetic intensity is solved into a series solution in terms of similarity parameters with the help of perturbation theory and MAPLE. The series is then analyzed to show the convergence of critical values and the change in bifurcation graph for Re and $\alpha$ by the positive effect of $\mathrm{Ha}$ with the help of Approximation method (Khan, 2002, Khan et al., 2003 and Rahman, 2004). In our analysis, it is found that the results are more accurate and uniform in comparison with Makinde (2006). The critical relationship among the parameters, an extension of Makinde (2006), is also shown graphically.

\section{Review of Pade'-Hermite approximants}

In 1893, Pade' and Hermite introduced Pade'-Hermite class. All the one variable approximants that were used or discussed throughout this paper belong to the Pade'-Hermite class. In its most general form, this class is concerned with the simultaneous approximation of several independent series.

Let $d \in \mathrm{N}$ and let the $d+1$ power series $U_{0}(x), U_{1}(x), \ldots, U_{d}(x)$ are given.

Assume that the $(d+1)$ tuple of polynomials $P_{N}^{[0]}, P_{N}^{[1]}, \ldots, P_{N}^{[d]}$

where $\quad \operatorname{deg} P_{N}^{[0]}+\operatorname{deg} P_{N}^{[1]}+\ldots+\operatorname{deg} P_{N}^{[d]}+d=N$,

is a Pade'-Hermite form of these series if

$$
\sum_{i=0}^{d} P_{N}^{[i]}(x) U_{i}(x)=O\left(x^{N}\right) \text { as } x \rightarrow 0 .
$$

Here $U_{0}(x), U_{1}(x), \ldots, U_{d}(x)$ may be independent series or different form of a unique series. We need to find the polynomials $P_{N}^{[i]}$ that satisfy the Equations (1) and (2). These polynomials are completely determined by their coefficients. So, the total number of unknowns in Equation (2) is

$$
\sum_{i=0}^{d} \operatorname{deg} P_{N}^{[i]}+d+1=N+1
$$

Critical behaviour of the MHD flow in convergent-divergent channels 
Expanding the left hand side of Equation (2) in powers of $x$ and equating the first $N$ equations of the system equal to zero, we get a system of linear homogeneous equations. To calculate the coefficients of the Pade'Hermite polynomials it require some sort of normalization, such as

$$
P_{N}^{[i]}(0)=1 \text { for some } 0 \leq i \leq d
$$

It is important to emphasize that the only input required for the calculation of the Pade'-Hermite polynomials are the first $N$ coefficients of the series $U_{0}, \ldots, U_{d}$. The equation (2.3) simply ensures that the coefficient matrix associated with the system is square. One way to construct the Pade'-Hermite polynomials is to solve the system of linear equations by any standard method such as Gaussian elimination or Gauss-Jordan elimination.

Drazin -Tourigney Approximants (2003) is a particular kind of algebraic approximants and Khan (2002) introduced High-order differential approximant as a special type of differential approximants. High-order partial differential approximants discussed in Rahman (2004) is a multivariable differential approximants. An algebraic programming language Maple available on www.maplesoft.com was used to compute the series coefficients of non-dimensional governing equation of the problem.

\section{Mathematical Formulation}

The time-independent two-dimensional flow from a source or sink at the intersection between two rigid plane walls of a viscous incompressible conducting fluid in presence of an external homogeneous magnetic field (Fig. 1 ) is considered. The small electrical conductivity of the fluid and the produced very small electromagnetic force are considered. Let $(r, \theta)$ be polar coordinate with $r=0$ as the sink or source and $\alpha$ be the semi-angle where the domain of the flow be $-|\alpha|<\theta<|\alpha|$.

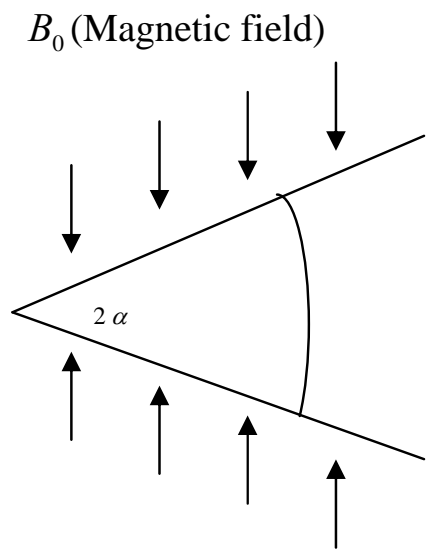

Fig. 1: Convergent-Divergent Channels

The Navier-Stokes equations in terms of the vorticity $(\omega)$ and stream-function $(\psi)$ can be written as Makinde (2006)

$$
\frac{1}{r} \frac{\partial(\psi, \omega)}{\partial(\theta, r)}=v \nabla^{2} \omega-\frac{\sigma_{e} B_{0}^{2}}{\rho r^{2}} \omega, \quad \omega=-\nabla^{2} \psi
$$

where, $\quad \nabla^{2}=\frac{\partial^{2}}{\partial r^{2}}+\frac{\partial}{r \partial r}+\frac{\partial^{2}}{r^{2} \partial \theta^{2}}$

with the boundary conditions

$$
\psi=\frac{Q}{2}, \quad \frac{\partial \psi}{\partial \theta}=0, \quad \text { at } \theta= \pm \alpha
$$

where the volumetric flow rate $Q=\int_{-\alpha}^{\alpha} u r d \theta$. A purely symmetrical radial flow is considered in Makinde (2006) for which the tangential velocity $v=0$ 
and the stream-function is defined by $\psi=\frac{Q G(\theta)}{2}$.

The non-dimensional governing equation and boundary conditions are obtained as:

$$
\frac{d^{4} G}{d \eta^{4}}+2 \operatorname{Re} \alpha \frac{d G}{d \eta} \frac{d^{2} G}{d \eta^{2}}+(4-H a) \alpha^{2} \frac{d^{2} G}{d \eta^{2}}=0
$$

with

$$
G=1, \quad \frac{d G}{d \eta}=0, \quad \text { at } \eta= \pm 1
$$

where, $\eta=\frac{\theta}{\alpha}, H a=\sqrt{\frac{\sigma B_{0}{ }^{2}}{\rho v}}$ is the magnetic parameter and $R e=\frac{Q}{2 v}$ is the flow Reynolds number.

Since Eq (7) is non-linear for $\alpha$, a series is considered in the form

$$
G(\eta)=\sum_{i=0}^{\infty} G_{i} \alpha^{i}
$$

We then find that $G(\eta)$ has a singularity at $\alpha=\alpha_{c}$ of the form

$$
G(\eta) \sim C\left(\alpha_{c}-\alpha\right)^{\beta_{c}}
$$

with the critical exponent $\beta_{c}$.

The non-dimensional governing equation is then solved in a series solution by substituting the Eq. (9) into Eq.(7) and equating the coefficients of powers of $\alpha$. With the help of MAPLE, we have computed the first 18 coefficients for the series of the stream function $G$ in terms of $\alpha, R e, H a$. The first few coefficients of the series for $G$ are

$$
\begin{aligned}
& G(\eta ; \alpha, R e, H a)=\frac{1}{2} \eta\left(3-\eta^{2}\right)-\frac{3}{280} \eta\left(\eta^{2}-5\right)(\eta-1)^{2}(\eta+1)^{2} \alpha R e- \\
& \frac{1}{431200} \eta\left(98 \eta^{6}-959 \eta^{4}+2472 \eta^{2}-2875\right)(\eta-1)^{2}(\eta+1)^{2} \alpha^{2} R e^{2}+ \\
& \frac{1}{40} \eta(\eta-1)^{2}(\eta+1)^{2}(4-H a) \alpha^{2}+
\end{aligned}
$$

Although the computational complexity increases rapidly, we managed to compute the first 75 terms for $G$ in terms of single parameter $\alpha$ for $H a=0,1, \ldots \ldots \ldots . .5$ at $R e=20$.

The first 75 terms for $G$ in terms of single parameter $R e$ for $H a=4$ at $\alpha=0.1$ is also computed. These series are then analyzed by differential and algebraic approximate methods to determine the critical values and bifurcation graphs of the channel angular width and flow Reynolds number for different values of magnetic parameter. The critical relationships among the parameters in the series are also shown graphically using partial differential approximate method.

\section{Results and Discussion}

The series in powers of $\alpha, R e$ and $H a$ in the following functional form proportional to the velocity of the flow along the centre line is considered for the investigation:

$$
G^{\prime}(\eta=0 ; \alpha, \operatorname{Re}, H a)
$$

Applying the differential approximation method into the single series of $\alpha$ and the series (11), the convergence of the critical value $\alpha_{C}$ with critical exponent $\beta_{c}$ for a wide range of magnetic parameter has been computed more significantly.

Table 1-6 display the convergence of $\alpha_{C}$ for different values of $H a(=0,1,2,3,4,5)$ with $R e=20$. The critical values are determined using both the single series of $\alpha$ and the series described in (11). It is seen that the values of $\alpha_{C}$ converges up to 26 decimal places at $d=10$ using $N=75$ terms of the single series of $\alpha$ . The values of $\alpha_{C}$ obtained by single series of $\alpha$ and the series (11) involving three parameters are observed equal at $d=4$ with $N=18$. It can be also noted that the critical value $\alpha_{C}$ increases uniformly for the increasing Hartman number. Moreover, in Table 1-6 the values of $\beta_{c}$ confirm that $\alpha_{C}$ is a branch point. 
Furthermore, Table.1 shows an excellent agreement at $\alpha_{C} \approx .2679608310828466960444352$ with the classical Jeffery-Hamel flows in absence of magnetic field. Finally, Table 7 interprets the comparison of our results with Makinde (2006) remarking that the variation of $\alpha_{C}$ due to the increase of magnetic parameter $H a$ is more accurate and uniform than Makinde (2006). The single series of $R e$ is used to show the convergence of critical value $\operatorname{Re}_{c}$ with critical exponent $\beta_{c}$ for $H a=4$ at $\alpha=0.1$. Table 8 estimates the convergence of $\operatorname{Re}_{c}$ up to 24 decimal places at $d=10$ using $N=75$. Also, in Table 8 the values of $\beta_{c}$ confirm that $\mathrm{Re}_{\mathrm{c}}$ is a branch point.

Table 1: Estimates of critical angles $\alpha_{C}$ and corresponding exponent $\beta_{c}$ at Re $=20$ and $H a=0$ using Highorder differential approximants (2002).

\begin{tabular}{|c|c|c|c|c|}
\hline $\mathrm{d}$ & $\mathrm{N}$ & $\alpha_{C}$ (single series) & $\alpha_{C}$ [ series (7)]) & \multicolumn{1}{c|}{$\beta_{c}$} \\
\hline 2 & 7 & .2749980919548527561596138 & .2749980919548527561596138 & .10878684389230764954 \\
\hline 3 & 12 & .2602059979752087905452916 & .2602059979752087905452916 & 1.6592008206154575273 \\
\hline 4 & 18 & .2679736610354297291067892 & .2679736610354297291067892 & .4953933331461112855 \\
\hline 5 & 25 & .2679607925653470037213470 & & .5000238205405836379 \\
\hline 6 & 33 & .2679608309216671706497074 & & .5000002225349214361 \\
\hline 7 & 42 & .2679608310828499910588531 & & .4999999999938879389 \\
\hline 8 & 52 & .2679608310828466960601169 & & .4999999999999999565 \\
\hline 9 & 63 & .2679608310828466960444344 & & .5000000000000000072 \\
\hline 10 & 75 & .2679608310828466960444352 & & .5000000000000000002 \\
\hline
\end{tabular}

Table 2: Estimates of critical angles $\alpha_{C}$ and corresponding exponent $\beta_{c}$ at $R e=20$ and $H a=1$ using High-order differential approximants (2002).

\begin{tabular}{|c|c|c|c|c|}
\hline $\mathrm{d}$ & $\mathrm{N}$ & $\alpha_{C}$ (single series) & $\alpha_{C}[$ series (7)] & $\beta_{c}$ \\
\hline 2 & 7 & .27665112580188032650165569 & .276651125801880326501655691 & 7.429197021826671482 \\
\hline 3 & 12 & .27176980615626427060569929 & .271769806156264270605699299 & -.261785713430648567 \\
\hline 4 & 18 & .26925148848575837254505344 & .2692514884857558372545053443 & .4561906332084395651 \\
\hline 5 & 25 & .26916241929088580028156064 & & .5000247289730165476 \\
\hline 6 & 33 & .26916245977114395877256265 & & .4999999906897554027 \\
\hline 7 & 42 & .26916245976318174485150479 & & .4999999999573921934 \\
\hline 8 & 52 & .26916245976315907745130293 & & .4999999999999998382 \\
\hline 9 & 63 & .26916245976315907739346092 & & .5000000000000000050 \\
\hline 10 & 75 & .26916245976315907739346096 & & .4999999999999999923 \\
\hline
\end{tabular}

Table 3: Estimates of critical angles $\alpha_{C}$ and corresponding exponent $\beta_{c}$ at $R e=20$ and $H a=2$ using High-order differential approximants (2002).

\begin{tabular}{|c|c|c|c|c|}
\hline $\mathrm{d}$ & $\mathrm{N}$ & $\alpha_{C}$ (single series) & $\alpha_{C}$ [ series (7)] & $\beta_{c}$ \\
\hline 2 & 7 & .27823197900192240193407708 & .27823197900192240193407708 & .5919848362542434934 \\
\hline 3 & 12 & .27379563943913053775199571 & .27379563943913053775199571 & .5448269070920006607 \\
\hline 4 & 18 & .27037606595782002309656470 & .27037606595782002309656470 & .5033015308595404481 \\
\hline 5 & 25 & .27038644263339964600928911 & & .5000555023997966286 \\
\hline 6 & 33 & .27038655906469678677030278 & & .4999999902052514669 \\
\hline 7 & 42 & .27038655905619460460995491 & & .4999999999975450022 \\
\hline 8 & 52 & .27038655905619320425304318 & & .4999999999999999820 \\
\hline 9 & 63 & .27038655905619320424824461 & & .4999999999999999990 \\
\hline 10 & 75 & .27038655905619320424824455 & & .5000000000000000029 \\
\hline
\end{tabular}


Table 4: Estimates of critical angles $\alpha_{C}$ and corresponding exponent $\beta_{c}$ at $R e=20$ and $H a=3$ using Highorder differential approximants (2002).

\begin{tabular}{|c|c|c|c|c|}
\hline $\mathrm{d}$ & $\mathrm{N}$ & $\alpha_{C}$ (single series) & $\alpha_{C}[$ series (7)] & $\beta_{c}$ \\
\hline 2 & 7 & .279762036762114265515350954 & .279762036762114265515350954 & .5322217290273408261 \\
\hline 3 & 12 & .269213999914381062053064886 & .269213999914381062053064886 & .4914784750977411087 \\
\hline 4 & 18 & .271630281415858951935860527 & .271630281415858951935860527 & .5011909765497291434 \\
\hline 5 & 25 & .271633941351507479960053773 & & .4999952474512750814 \\
\hline 6 & 33 & .271633934182549283009691130 & & .49999999973031274357 \\
\hline 7 & 42 & .271633934180195495771995551 & & .5000000000038244847 \\
\hline 8 & 52 & .271633934180197600883162104 & & .5000000000000000062 \\
\hline 9 & 63 & .271633934180197600883506790 & & .49999999999999999971 \\
\hline 10 & 75 & .271633934180197600883506774 & & .4999999999999999913 \\
\hline
\end{tabular}

Table 5: Estimates of critical angles $\alpha_{C}$ and corresponding exponent $\beta_{c}$ at $R e=20$ and $H a=4$ using High-order differential approximants (2002).

\begin{tabular}{|c|c|c|c|c|}
\hline $\mathrm{d}$ & $\mathrm{N}$ & $\alpha_{C}$ (single series) & $\alpha_{C}$ [series (7)] & $\beta_{c}$ \\
\hline 2 & 7 & .28125951132635432681080696 & .2812595113263543268108069639 & .533349118995105506 \\
\hline 3 & 12 & .27226274077853037159610100 & .2722627407785303715961010067 & .498228540542405286 \\
\hline 4 & 18 & .27290283574099710569184306 & .2729028357409971056918430660 & .500847691647703434 \\
\hline 5 & 25 & .27290544292488388754260176 & & .499994428166863026 \\
\hline 6 & 33 & .27290543430380172571596594 & & .500000001972260338 \\
\hline 7 & 42 & .27290543430555951905919161 & & .5000000000001361310 \\
\hline 8 & 52 & .27290543430555959319476103 & & .499999999999999997 \\
\hline 9 & 63 & .27290543430555959319333598 & & .5000000000000000115 \\
\hline 10 & 75 & .27290543430555959319333598 & & .5000000000000000100 \\
\hline
\end{tabular}

Table 6: Estimates of critical angles $\alpha_{C}$ and corresponding exponent $\beta_{c}$ at $R e=20$ and $H a=5$ using High-order differential approximants (2002).

\begin{tabular}{|c|c|c|c|c|}
\hline $\mathrm{d}$ & $\mathrm{N}$ & $\alpha_{C}$ (single series) & $\alpha_{C}$ [ series (7)] & $\beta_{c}$ \\
\hline 2 & 7 & .282740455470542194962772877 & .28274045547054219496277 & .5316268843464719954 \\
\hline 3 & 12 & .273920667962368822833379792 & .27392066796236882283337 & .4997345430256748736 \\
\hline 4 & 18 & .274199503848608338641744612 & .27419950384860833864174 & .5007915959995632061 \\
\hline 5 & 25 & .274201956842852361389389378 & & .4999993169363610355 \\
\hline 6 & 33 & .274201955830691574449370731 & & .5000000014203295731 \\
\hline 7 & 42 & .274201955831973233791395193 & & .4999999999998953730 \\
\hline 8 & 52 & .274201955831973177295547888 & & .5000000000000000020 \\
\hline 9 & 63 & .274201955831973177293986707 & & .49999999999999999943 \\
\hline 10 & 75 & .274201955831973177293986712 & & .4999999999999999865 \\
\hline
\end{tabular}

The positive change in $R e_{c}$ with the increasing intensity of $H a$ is shown in Table 9 more accurately and significantly in comparison with Makinde (2006). As the Jeffery-Hamel flow (absence of magnetic field) $\mathrm{Re}_{c} \approx 54.58108686111191863866719680$ is obtained that is consistent with Fraenkel's (1962) asymptotic result, $\operatorname{Re}_{c} \sim \frac{5.461}{\alpha}$ as $\alpha \rightarrow 0$, when $H a=0$. However, the calculation by Khan (2002) shows that $\operatorname{Re}_{c} \sim \frac{5.4581086861111918638}{\alpha}$ as $\alpha \rightarrow 0$. 
Table 7: Comparisons of critical angles $\alpha_{C}$ and corresponding critical exponent $\beta_{c}$ at $\operatorname{Re}=20$ using High-order differential approximants (2002). The result is comparable with the result of Makinde (2006).

\begin{tabular}{|c|c|c|c|c|c|c|c|}
\hline \multirow{3}{*}{$\begin{array}{l}\begin{array}{l}\text { HODA } \\
d=10 \\
N=75\end{array}\end{array}$} & $\mathrm{Ha}$ & 0 & 1 & 2 & 3 & 4 & 5 \\
\hline & $\alpha_{C}$ & $\begin{array}{c}.267960831 \\
0828466960 \\
444352\end{array}$ & $\begin{array}{c}.26916245 \\
97631590 \\
77393460 \\
96\end{array}$ & $\begin{array}{c}.270386559 \\
0561932042 \\
4824455\end{array}$ & $\begin{array}{c}.271633934 \\
1801976008 \\
83506774\end{array}$ & $\begin{array}{c}.2729054343 \\
05559593193 \\
33598\end{array}$ & $\begin{array}{c}.274201955 \\
8319731772 \\
93986712\end{array}$ \\
\hline & $\beta_{c}$ & $\begin{array}{r}.500000000 \\
0000000002\end{array}$ & $\begin{array}{c}.49999999 \\
99999999 \\
92\end{array}$ & $\begin{array}{l}.500000000 \\
0000000029\end{array}$ & $\begin{array}{l}.4999999999 \\
9999999913\end{array}$ & $\begin{array}{c}.5000000000 \\
000000100\end{array}$ & $\begin{array}{l}.499999999 \\
9999999865\end{array}$ \\
\hline \multirow{3}{*}{$\begin{array}{l}\text { Makind } \\
\text { e } \\
(2006)\end{array}$} & $H a$ & 0 & 1 & 2 & 3 & 4 & 5 \\
\hline & $\alpha_{C}$ & 0.267960 & 0.269162 & 0.272906 & 0.279878 & 0.290431 & 0.307406 \\
\hline & $\beta_{c}$ & 0.50000 & 0.50000 & 0.50000 & 0.50000 & 0.50000 & 0.50000 \\
\hline
\end{tabular}

Table 8: Estimates of critical Reynolds numbers $\mathrm{Re}_{\mathrm{c}}$ and corresponding exponent $\beta_{c}$ at $\alpha=0.1$ and $H a=4$ using High-order differential approximants (2002).

\begin{tabular}{|c|c|c|c|}
\hline $\mathrm{d}$ & $\mathrm{N}$ & $\operatorname{Re}_{c}$ & $\beta_{c}$ \\
\hline 2 & 7 & 56.25190226527086536216139279 & .7209818858072179797 \\
\hline 3 & 12 & 54.45254815570607431922020134 & .4841068361716114107 \\
\hline 4 & 18 & 54.58056714819942113836861321 & .4999296570680748621 \\
\hline 5 & 25 & 54.58108858497677750852035270 & .4999944281668630267 \\
\hline 6 & 33 & 54.58108686076034514319318997 & .5000000019722603390 \\
\hline 7 & 42 & 54.58108686111190381183832271 & .5000000000001361302 \\
\hline 8 & 52 & 54.58108686111191863895220716 & .4999999999999999939 \\
\hline 9 & 63 & 54.58108686111191863866719641 & .4999999999999999998 \\
\hline 10 & 75 & 54.58108686111191863866719680 & .4999999999999999953 \\
\hline
\end{tabular}

Table 9: Comparisons of critical Reynolds number $R e_{C}$ and corresponding exponent $\beta_{c}$ at $\alpha=0.1$ using High-order differential approximants (2002) and Makinde (2006).

\begin{tabular}{|c|c|c|c|c|c|c|c|}
\hline $\begin{array}{c}\text { HODA } \\
\mathrm{D}=4 \\
\mathrm{~N}=8\end{array}$ & $H a$ & 0 & 1 & 2 & \multicolumn{1}{c|}{3} & 4 & 5 \\
\cline { 2 - 8 } & $\mathrm{Re}_{\mathrm{c}}$ & 54.44407939 & 54.47805874 & 54.51340970 & 54.54702585 & 54.58135150 & 54.61676356 \\
\cline { 2 - 8 } & $\beta_{c}$ & .4991155356 & .4991155356 & .4991155356 & .4997069458 & .5001231984 & .4985944495 \\
\hline \multirow{2}{*}{$\begin{array}{l}\text { Makinde } \\
(2006)\end{array}$} & $H a$ & 0 & 1 & 2 & 3 & 4 & 5 \\
\cline { 2 - 8 } & $\mathrm{Re}_{c}$ & 54.4389 & 54.47179 & 54.58087 & 54.66510 & 55.22071 & 55.52727 \\
\cline { 2 - 8 } & $\beta_{c}$ & 0.50000 & 0.50000 & 0.50000 & 0.50000 & 0.50000 & 0.50000 \\
\hline
\end{tabular}

Fig. 2 illustrates the effect of varying values of magnetic parameter $H a(=0,5)$ on the bifurcation graph of $\alpha_{C}$ at $\operatorname{Re}=20$. Here it is seen that the bifurcating point $\alpha_{C}$ changes clearly from $0.26797366(H a=0)$ to $0.27419950(H a=5)$.

Fig. 3(a) and Fig. 3(b) show how variations of $\mathrm{Ha}$ affect the flow. It can be noted that an increase in the values of $H a$ leads to a change in the bifurcation graph of critical Reynolds number at $\alpha=0$. The bifurcating point $\mathrm{Re}_{\mathrm{c}}$ changes from $54.44407939(H a=0)$ to $54.61676356(H a=5)$ shown sharply in large scale in Fig. 3(b). 
There it is clearly seen that the positive effect of magnetic intensity changes the behaviour of the nondimensional flow parameter as a result the solution behaviour.

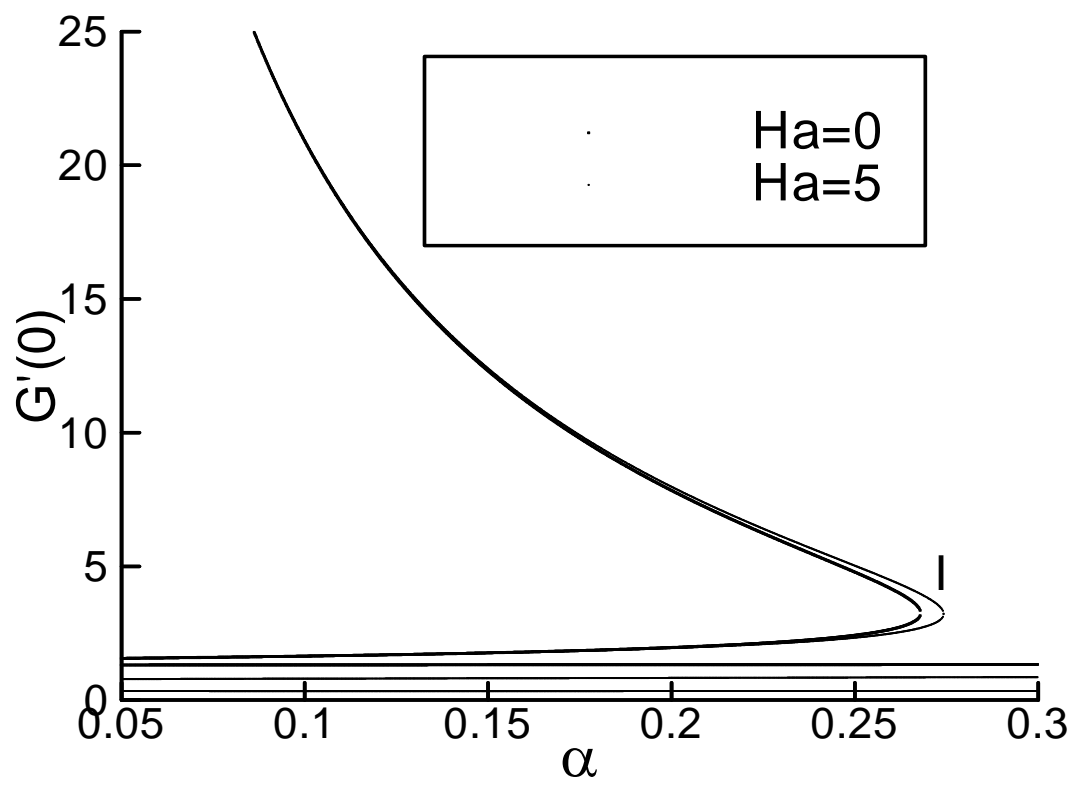

Fig. 2: Approximate bifurcation diagram (curve I) of $\alpha_{c}$ in the $\left(\alpha, G^{\prime}(0)\right.$ ) Plane with $H a=0$ and $H a=5$ obtained by Drazin-Tourigny method (2003) for $d=8$. The other curves are spurious.

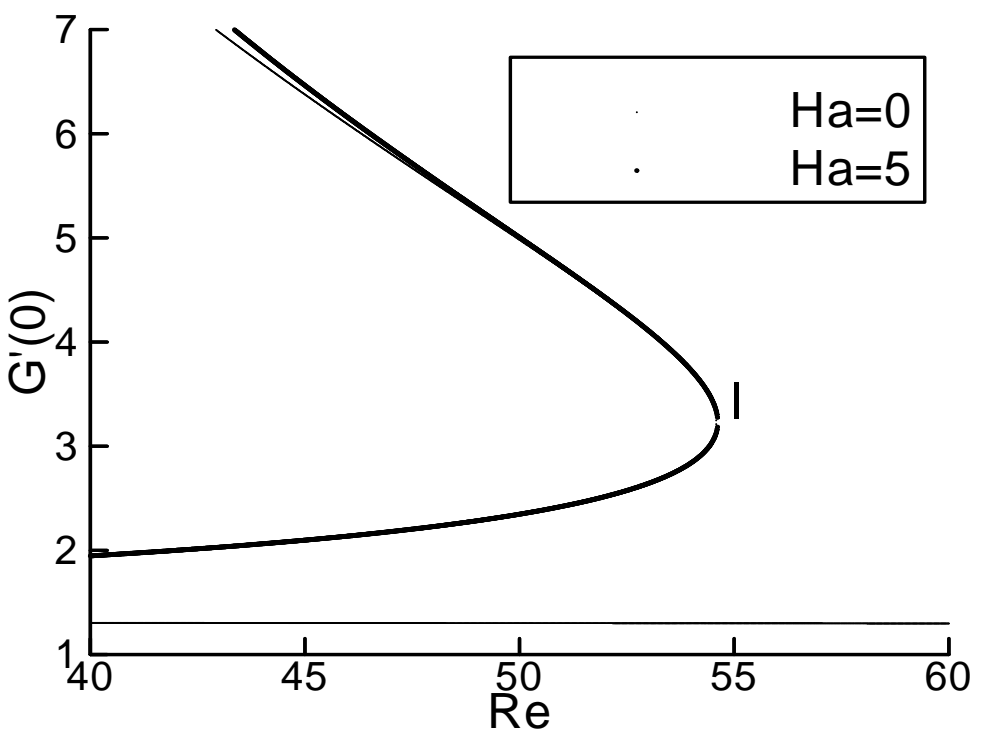

Fig. 3 (a): Approximate bifurcation diagram (curve I) of $\operatorname{Re}_{c}$ in the $\left(\mathrm{Re}, G^{\prime}(0)\right.$ ) Plane with $H a=0$ and $H a=5$ obtained by Drazin-Tourigny method (2003) for $d=4$. The other curve is spurious. 


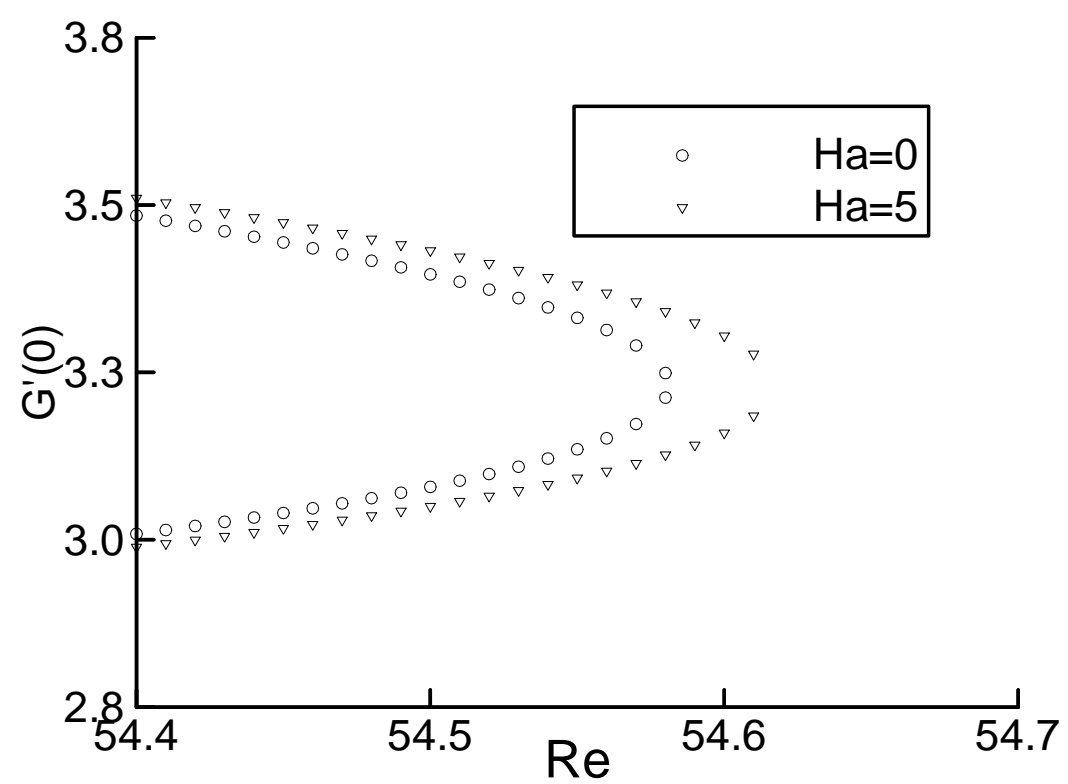

Fig. 3(b): Approximate bifurcation diagram (curve I) of $\operatorname{Re}_{c}$ in the $\left(\mathrm{Re}, G^{\prime}(0)\right.$ ) Plane with $H a=0$ and

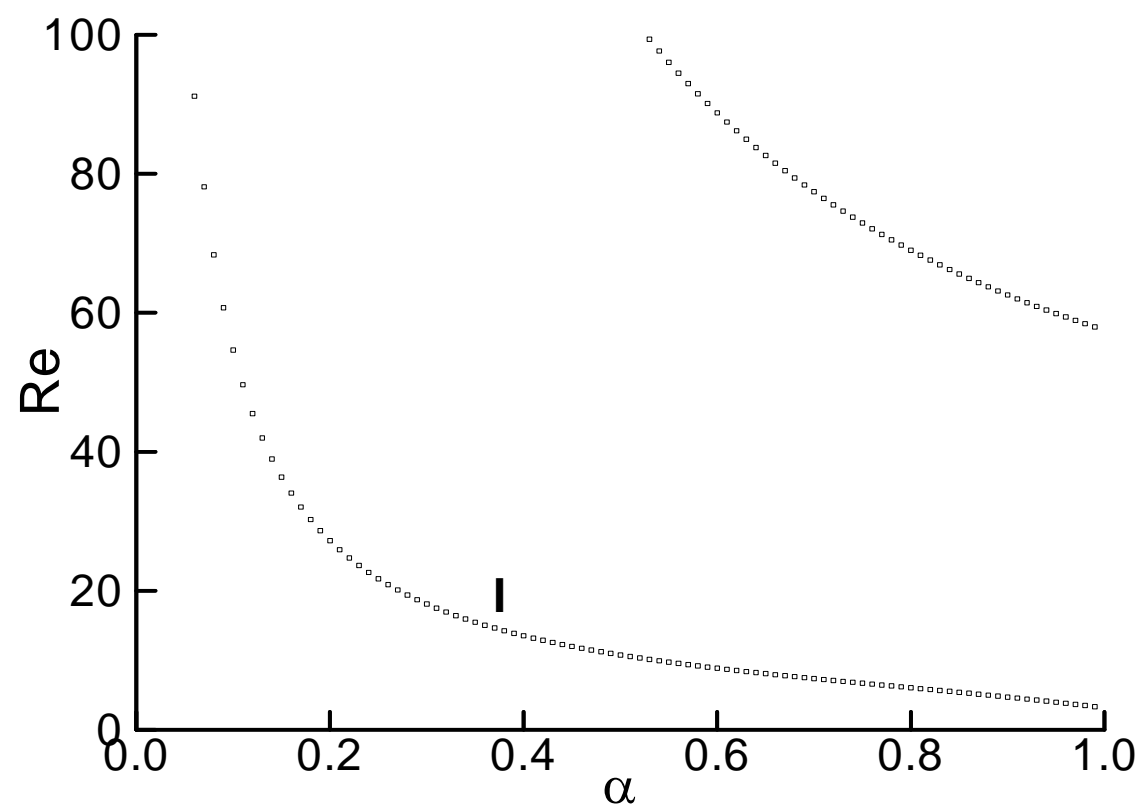

Fig. 4: Critical $\alpha$-Re relationship (curve I) using High-order partial Differential approximants (2004) with $d=6$. The other curve is spurious. 


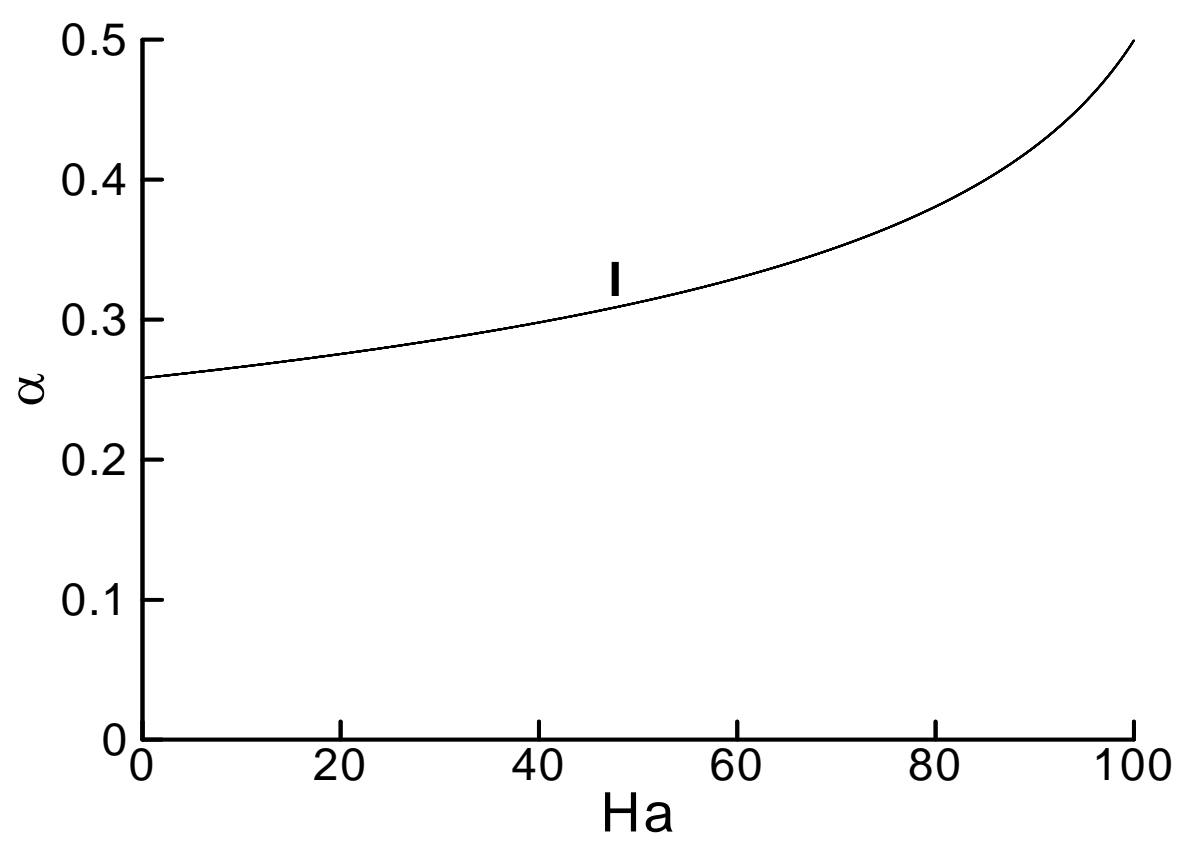

Fig. 5: Critical $\mathrm{Ha}-\alpha$ relationship (curve I) using High-order partial Differential approximants (2004)

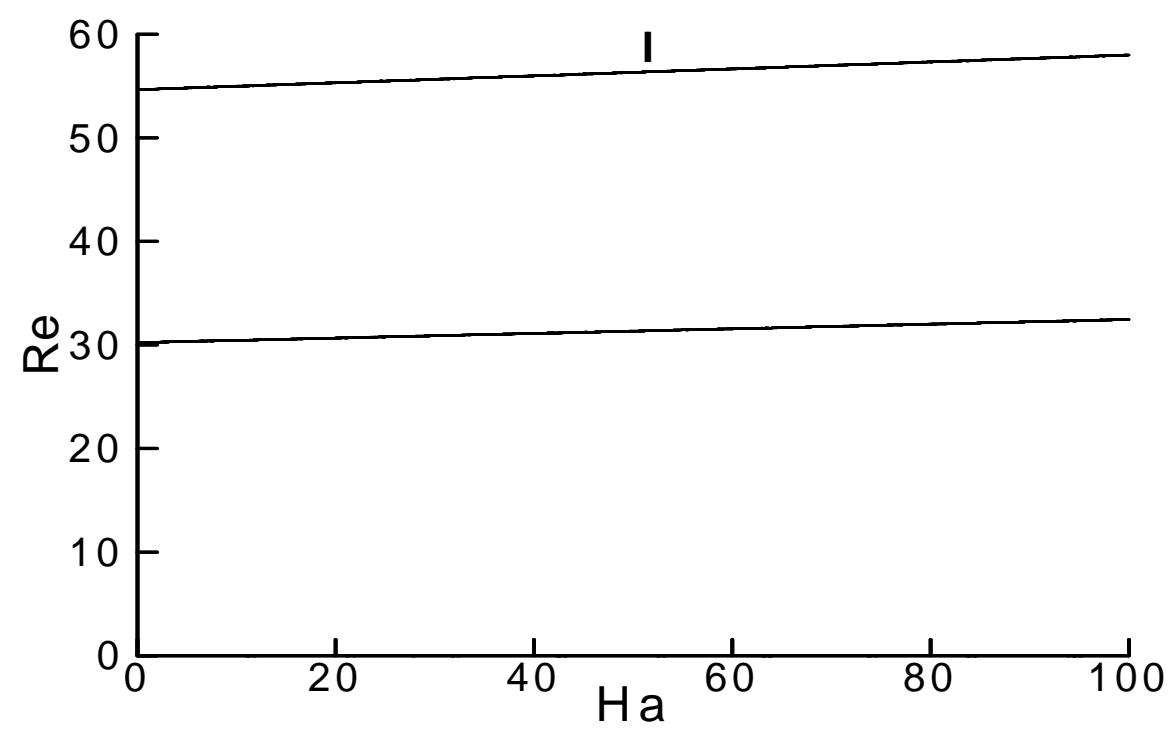

Fig. 6: Critical $H a-$ Re relationship (curve I) using High-order partial Differential approximants (2004) with $d=6$. The other curve is spurious.

The High-order Partial Differential Approximant (2004) is applied to the series (11) in order to determine the critical relationship among the parameters $\alpha$, Re, Ha. Fig. 4 displays the critical relation between the channel angular width $\alpha$ and Reynolds number Re. It is found that as $\alpha$ increases then Re decreases and conversely Re increases when $\alpha$ decreases. This implies that both channel angle and Reynolds number are inversely proportional to each other. From Fig. 5 it can be observed that the magnetic parameter increases the channel 
angular width $\alpha$ gradually that is an excellent agreement with the numerical values in Table 7. Fig. 6 represents clearly that the Reynolds number Re increases slowly for increasing Ha The conjecture of the figure exactly supports the numerical solution in Table 9 .

\section{Conclusion}

In this paper, we have used power series to study the critical behaviour of the time independent, twodimensional, laminar flow through Convergent-Divergent Channels in the presence of a magnetic field.

- By exploiting various generalizations of the Pade'-Hermite approximations, we have obtained accurate numerical approximations of the critical parameters of the flow, which involves physically the instability of the problem.

- Besides Makinde's (2006) result, our finding is the critical relationship among the parameters.

- Moreover, we provide a basis for guidance about new approximants idea for summing power series should be chosen for many problems in fluid mechanics and similar subjects. We elaborate this guidance here as reference.

- The computing costs of finding the coefficients of a power series of this problem are higher than the costs of processing them by a summation method. So it behoves the user to exploit all the available information about the problem that gives rise to the series.

By using High-order differential approximants we find more accurate result than those in Makinde (2006). Rapid convergence of summation, when it takes place, has given great confidence that the error is in fact small and that the method of summation chosen not only given accurate numerical results, but also gives the asymptotic form of the singularity beyond reasonable doubt.

\section{References}

Banks, W. H. H., Drazin, P. G., and Zaturska, M. B. (1988): On perturbation of Jeffery-Hamel flow, Journal of Fluid Mechanics., Vol. 186, pp. 559-581. doi:10.1017/S0022112088000278

Fraenkel, L. E. (1962): Laminar flow in symmetrical channels with slightly curved walls. I: On the JefferyHamel solutions for flow between plane walls, Proceedings of the Royal Society of London, Vol. 267, pp. 119138. doi:10.1098/rspa.1962.0087

Hamel, G., (1916): Spiralförmige Bewgungen Zäher Flüssigkeiten, Jahresbericht der Deutschen Math. Vereinigung, Vol. 25, pp. 34-60.

Jeffery, G. B., (1915): The two-dimensional steady motion of a viscous fluid, Philosophical Magazine, Vol. 6, pp. 455-465.

Khan, M.A.H., (2002): High-Order Differential Approximants, Journal of Computational. and Applied Mathematics, Vol. 149, pp. 457-468. doi:10.1016/S0377-0427(02)00561-7

Khan, M.A.H., Drazin, P. G., and Tourigny, Y., (2003): The summation of series in several variable and its applications in fluid dynamics, Fluid Dynamics Research, Vol. 33, pp. 191-205. doi:10.1016/S01695983(03)00038-8

Kayvan, S., Navid K., and Seyed-Mohammad, T.(2007): Magnetohydrodynamic (MHD) flows of viscoelastic fluids in converging/diverging channels, International Journal of Engineering Science, Vol.45, No.11, pp. 923938.

Makinde, O. D., (1997): Steady flow in a linearly diverging asymmetrical channel, Computer Assisted Mechanics and Engineering Sciences, Vol. 4, pp. 157-165.

Makinde, O. D., (2006): Hermite-Pade' Approximation approach to Hydromagnetic flows in convergentdivergent channels, Applied Mathematics and Computation, Vol. 181, No. 2, pp. 966-972.

doi:10.1016/j.amc.2006.02.018

Makinde, O. D., (2008): Effect of arbitrary magnetic Reynolds number on MHD flows in convergent-divergent channels, International Journal of Numerical Methods for Heat \& Fluid Flow, Vol.18, No.6, pp. 697-707 doi:10.1108/09615530810885524

Rahman, M.M., (2004): A New Approach to Partial Differential Approximants, M. Phil, BUET, Dhaka.

Sobey, I. J., and Drazin, P. G., (1986): Bifurcations of two-dimensional channel flows, Journal of Fluid Mechanics., Vol. 171, pp. 263-287. doi:10.1017/S0022112086001441 